\title{
Influence of some parameters on the ability of Listeria monocytogenes, Listeria innocua, and Escherichia coli to form biofilms
}

\author{
Sara Lezzoum-Atek ${ }^{1,2}$, Leila Bouayad ${ }^{1}$ and Taha Mossadak Hamdi ${ }^{1}$ \\ 1. Laboratory of Food Hygiene and Quality Insurance System, High National Veterinary School, Algiers, Algeria \\ 2.Biology Department, Faculty of Sciences of Nature and Life and Earth Sciences, University of Bouira, Algeria. \\ Corresponding author: Sara Lezzoum-Atek, e-mail: sara_lezzoum@hotmail.fr \\ Co-authors: LB: leila bouayad@hotmail.com, TMH: moussahamdi@hotmail.com \\ Received: 28-11-2018, Accepted: 11-02-2019, Published online: 26-03-2019
}

doi: 10.14202/vetworld.2019.459-465 How to cite this article: Lezzoum-Atek S, Bouayad L, Hamdi TM (2019) Influence of some parameters on the ability of Listeria monocytogenes, Listeria innocua, and Escherichia coli to form biofilms, Veterinary World, 12(3): 459-465.

\begin{abstract}
Aim: The present study was conducted to evaluate the capacity of Listeria monocytogenes (L.m), Listeria innocua (L.i), and Escherichia coli to form biofilms on polystyrene support under different parameters by performing crystal violet (CV) staining technique.

Materials and Methods: Different suspensions were prepared with single strains and with multiple combinations of strains including two serogroups of L.m (IIa and IIb), L.i, and E. coli strains at different microbial load. Selected strains and combinations were grown in biofilms for 6 days attached to polystyrene microplates under aerobic and microaerophilic conditions. The evaluation of the power of adhesion and biofilm formation was determined by CV staining followed by the measurement of optical density at $24 \mathrm{~h}, 72 \mathrm{~h}$, and 6 days incubation time with and without renewal of the culture medium.

Results: All the strains tested, presented more or less adhesion power depending on the variation of the studied parameters as well as the ability to form multispecies biofilms. Their development is more important by renewing the culture medium and increasing the initial load of bacteria. The ability to adhere and form biofilms differs from one serogroup to another within the same species. In bacterial combination, strains and species of bacteria adopt different behaviors.

Conclusion: The ability to form biofilms is a key factor in the persistence of tested strains in the environment. Our study showed that L.m, L.i, and E. coli could adhere to polystyrene and form biofilms under different conditions. More researches are necessary to understand the mechanisms of biofilm formation and the influence of different parameters in their development.
\end{abstract}

Keywords: biofilm, Escherichia coli, Listeria innocua, Listeria monocytogenes, polystyrene support, variation of parameters.

\section{Introduction}

Biofilms existence and formation are well documented for many years. Any solid surface in contact with fluid containing bacteria is likely to be a biofilm carrier. Some authors estimate that biofilms represent the lifestyle of $>99 \%$ of terrestrial bacteria [1]. Biofilms colonize various surfaces; they are particularly known for their adverse effects in the fields of public health and industry, their most known negative impacts are nosocomial diseases, contamination of food products, and biodeterioration of materials, particularly biocorrosion and biological fouling of industrial equipment [2]. A biofilm is defined as a community of microorganisms adhering to a surface and surrounded by a complex matrix of exopolysaccharides (EPSs) made of substances synthesized by these same microorganisms $[3,4]$. Its composition is

Copyright: Lezzoum-Atek, et al. Open Access. This article is distributed under the terms of the Creative Commons Attribution 4.0 International License (http://creativecommons.org/licenses/ by/4.0/), which permits unrestricted use, distribution, and reproduction in any medium, provided you give appropriate credit to the original author(s) and the source, provide a link to the Creative Commons license, and indicate if changes were made. The Creative Commons Public Domain Dedication waiver (http:// creativecommons.org/publicdomain/zero/1.0/) applies to the data made available in this article, unless otherwise stated. carbohydrates, proteins, and other substances such as lipids or DNA [5]. The EPS provides protection to the microbial population of biofilms by concentrating nutrients, sequestering toxins, and preventing desiccation and biocides action [6]. The previous studies have confirmed the ability of different microbial species to form biofilms and persist in the environment, such as Salmonella, Staphylococcus [7,8], Listeria monocytogenes (L.m) [9], Listeria innocua (L.i) [10], Escherichia coli [11], and Campylobacter jejuni [12]. The ability of bacteria to form biofilms and their quantification was studied by applying different techniques including the use of polystyrene microplates [13] and crystal violet (CV) staining [14]. CV staining, originally described by Christensen et al. [15] and modified by Stepanovic et al. [16], allows the quantification of the biomass of a few hours or a few days old thin biofilms.

This study aimed to test the ability of L.m, L.i, and $E$. coli strains to form biofilms on 96-well polystyrene microplates by performing CV staining technique. Five influencing parameters were studied: Aerobic and microaerophilic conditions, microbial load, renewal of the culture medium, incubation time, and bacterial species composing the biofilm. 


\section{Materials and Methods}

\section{Ethical approval}

Ethical approval was not needed for this study.

\section{Bacterial strains}

The used strains of L.m and L.i were obtained from a previous study [17]. Six serogrouped and pulsotype strains of L.m were tested, among them, three strains (L.m 026, L.m 038, and L.m 055) belonged to the same serogroup IIa (L.m/IIa) and the same pulsotype; two strains (L.m 023 and L.m 043) to serogroup $I I a$, but each one to a different pulsotype and one strain (L.m 036) belonged to serogroup IIb (L.m/IIb) and had its pulsotype. E. coli strains were collected from surfaces in a slaughterhouse according to the ISO 18593 (2004) method [18]. Isolation and identification of these strains were carried out on chromID $^{\text {TM }}$ Coli agar (COLI ID-F) (bioMérieux) according to the recommendations of the ISO 4832 (2006) method [19]. The putative colonies were confirmed using the API 20E strip.

\section{Culture media and growing conditions}

The adhesion power and the ability of bacteria to form biofilm were carried out in two stages. The first one was to prepare different bacterial suspensions from different species and different strains, and the second one is to test the capacity of adhesion and biofilm formation of the different bacterial suspensions on polystyrene microplates by performing a $\mathrm{CV}$ staining (stain which binds to bacteria and EPS in the extracellular matrix of the biofilm) [20]. The bacteria were revivified by streaking onto Trypticase Soy Agar (bioMérieux) for $24 \mathrm{~h}$ at $37^{\circ} \mathrm{C}$ and enriched at $37^{\circ} \mathrm{C}$ for $24 \mathrm{~h}$ on Trypticase Soy Broth (TSB) (bioMérieux) to produce bacterial suspensions. The influence of the bacterial load, strains and species combinations and their concentrations on the ability of bacteria to adhere and form biofilms (single strain, single species, and multispecies) were investigated. On this purpose, bacterial suspensions were prepared with concentration adjusted to 1 and $4 \mathrm{McF}$ arland using a densitometer (DensiCHEK Plus Instrument, bioMérieux). The 1 McFarland bacterial suspensions were prepared from the single strains and from the following combinations: L.m/IIa, L.m/IIa+L.i, L.m/IIb+L.i, L.m/IIa+ E.coli, L.m $/ I I b+E$.coli, L.m. $/ I I a+L . i+E$. coli, L.m $/ I I b+$ L.i. $+E$. coli, and L.i+E. coli. The suspensions at $4 \mathrm{McFarland}$ were prepared from strains alone and from the following combinations: L.m $/ I I a+E$. coli, L.m $/ I I b+E$. coli, and L. $i+E$. coli. The obtained suspensions were seeded for each experimentation on eight triplicate polystyrene microplates. Measurements of the optical density (OD) at $550 \mathrm{~nm}$ were realized at $24 \mathrm{~h}$ to estimate the adhesion power. Two further readings were performed after $72 \mathrm{~h}$ and 6 days of incubation to estimate the ability of strains to form biofilms [21-23]. The method of assessing biofilm formation was based on the technique described by Stepanović et al. [16,24] and adapted for the analysis of selected strains and combinations. $200 \mu \mathrm{l}$ of each suspension were inoculated into triplicates; the last three columns were filled with $200 \mu$ of TSB to serve as controls. To test the effect of the oxygen content on the adhesion and the biofilms formation, four microplates were incubated in aerobic conditions while others in microaerophilic conditions using GENbox microaer and GENbag microaer (bioMérieux) for 6 days at $37^{\circ} \mathrm{C}$. The first reading of the ODs was performed after $24 \mathrm{~h}$ of incubation. One aerobic incubated plate and one microaerophilic incubated plate were retained to achieve a renewal of the culture medium to evaluate its effect on the development of biofilms. The wells were gently overturned and then filled with $200 \mu \mathrm{l}$ of TSB. The retained plates for this test were subsequently incubated with the other plates for $96 \mathrm{~h}$. Before measuring the OD, the wells were aspirated, rinsed with sterile physiological water 3 times, and fixed by adding $200 \mu$ of methanol to each well. After $15 \mathrm{~min}$, the plates were overturned, dried at ambient temperature, and then stained by adding $200 \mu \mathrm{l}$ of $2 \% \mathrm{CV}$ solution (bioMérieux). After $5 \mathrm{~min}$, the plates were rinsed with running water 5 times and dried at room temperature. The OD corresponding to each bacterial species, strains, and combination of species (ODs) was obtained by calculating the average of the triplicates, then compared with the averages of the ODs of the control wells (ODcn) [25]. The evaluation of the power of adhesion and biofilm formation is performed by applying the following classification: No biofilm production if ODs $\leq$ ODnc; low biofilm production when ODnc $<$ ODs $\leq 2$. ODnc; moderate $(\mathrm{M})$ production of biofilm 2 .ODnc $<$ ODs $\leq 4$. ODnc and strong (S) biofilm production 4.ODnc $<$ ODs [13,26,27].

\section{Statistical analysis}

The XLSTAT-Premium software was used to analyze the OD values collected by applying a oneway analysis of variance. The averages were considered significantly different for values of $\mathrm{p}<0.05$.

\section{Results}

\section{Formation of single-species biofilms}

In aerobic conditions and at $1 \mathrm{McF}$ arland concentration, all strains of L.m and L.i showed a strong adhesion after $24 \mathrm{~h}$ of incubation and a biofilm formation at $72 \mathrm{~h}$, except the strain L.m 038 (IIa) which showed a medium adhesion after $24 \mathrm{~h}$, then a strong biofilm production after $72 \mathrm{~h}$ and for the strain L.m 036 (IIb) which showed a medium adhesion after $24 \mathrm{~h}$ and a medium production of biofilm at $72 \mathrm{~h}$ and after 6 days (Table-1).

In microaerophilic conditions, all strains of L.m and L.i tested showed a medium adhesion after $24 \mathrm{~h}$ of incubation, followed by a strong biofilm production after $72 \mathrm{~h}$, except for the strain L.m 036 (IIb) which already showed a strong adhesion from the first $24 \mathrm{~h}$. Adhesion and biofilm formation of $E$. coli in aerobic or microaerophilic conditions were strong. 
Table-1: Results of adhesion and biofilm formation tests of L.m, L.i, and E. coli strain at 1 McFarland suspensions.

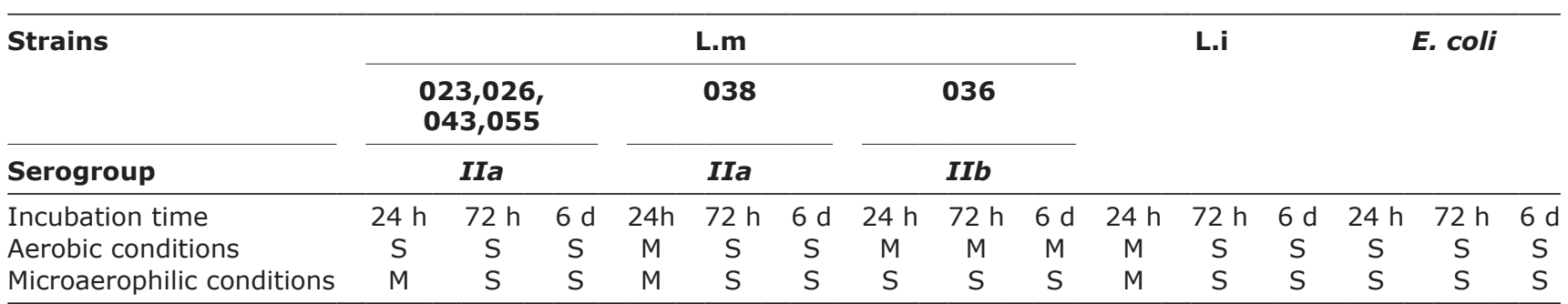

$\mathrm{S}=$ Strong, $\mathrm{M}=$ Moderate, $\mathrm{h}=$ Hours, $\mathrm{d}=$ Days, E. coli=Escherichia coli, L.m=Listeria monocytogenes, L. $\mathrm{i}=$ Listeria innocua

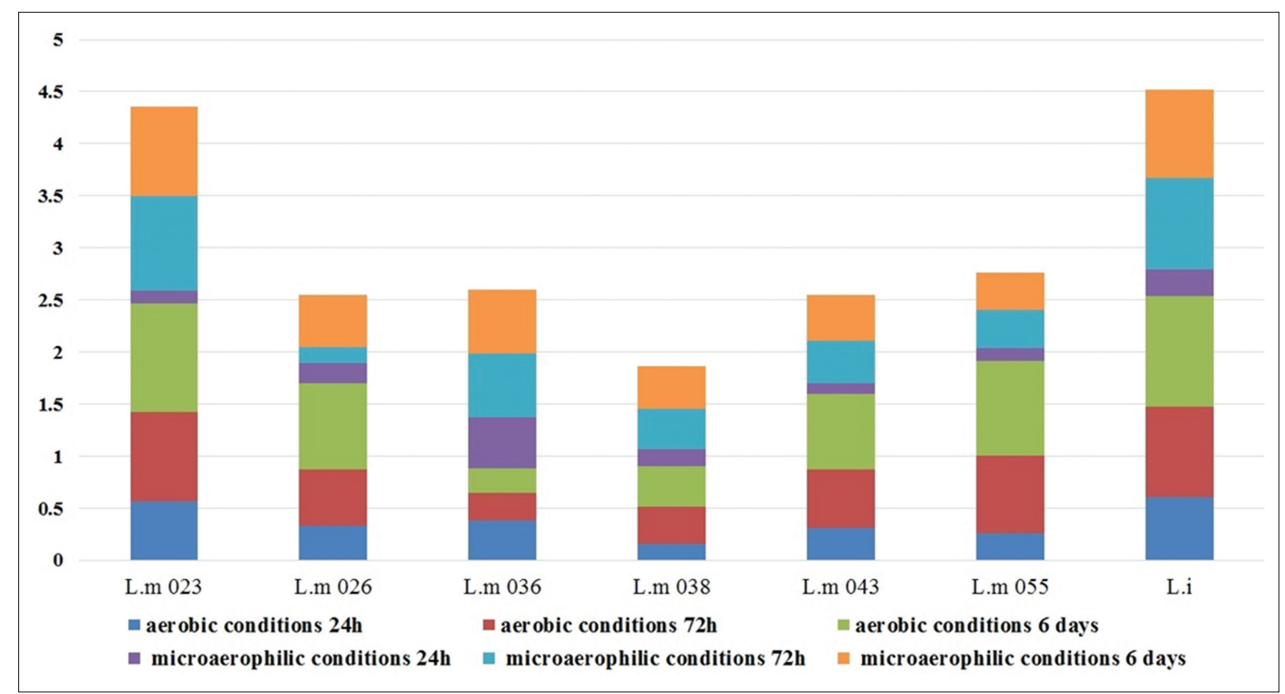

Figure-1: Effect of aerobic and microaerophilic conditions on biofilm formation from 1 McFarland suspensions. Captions: L.m: Listeria monocytogenes, L.i: Listeria innocua, OD: Optical density, h: Hour.

However, biofilms development was more important in microaerophilic than in aerobic conditions $(\mathrm{p}<0.05)$ (Figure-1).

At 4 McFarland concentration, all the strains of L.m, L.i, and E. coli showed a strong adhesion from the first $24 \mathrm{~h}$ of incubation and biofilms formation, in aerobic or microaerophilic conditions. The development of $E$. coli strains was higher in microaerophilic than in aerobic conditions $(\mathrm{p}<0.05)$.

\section{Formation of multistrain and multispecies biofilms}

In aerobic conditions, all stains combination tested at $1 \mathrm{McF}$ arland showed a strong adhesion from the first $24 \mathrm{~h}$ of incubation with a large formation of biofilm. In microaerophilic conditions, the adhesion was strong at $24 \mathrm{~h}$ for all the strain combination except for the combination of L.m 038+L.m 026+L.m 043 (L.m/IIa) which showed a moderate adhesion after $24 \mathrm{~h}$ of incubation and for the combination of L.m 023+L.m 026+Li (L.m/IIa+L.i) which showed a moderate adhesion and $\mathrm{M}$ biofilm production after $72 \mathrm{~h}$ of incubation. All combinations of bacterial strains tested at $4 \mathrm{McF}$ arland showed a strong adhesion from the first $24 \mathrm{~h}$ of incubation with a large biofilm formation in aerobic and microaerophilic conditions.

\section{Effect of the renewal of the culture medium}

Renewal of the culture medium on $1 \mathrm{McF}$ arland suspensions has given a higher development of biofilms in both aerobic and microaerophilic conditions.
OD measurements at $72 \mathrm{~h}$ of incubation with the renewal of the culture medium gave similar results to those obtained with the same strains after 6 days of incubation without renewal of the culture medium.

\section{Effect of incubation time}

Measurements of OD at $24 \mathrm{~h}, 72 \mathrm{~h}$, and 6 days showed that the development of biofilms increased significantly during the first $72 \mathrm{~h}$. This increase tends to stabilize during the followed $72 \mathrm{~h}$. The results of the OD measurements after 6 days of incubation showed a significant difference compared to those obtained after $72 \mathrm{~h}$ of incubation for most strains $(\mathrm{p}<0.05)$ (Figure-2).

\section{Discussion}

\section{Formation of single-species biofilms}

The previous studies have reported that species of the genus Listeria and the genus E. coli can form biofilms on different types of surfaces $[9,26,28]$. Our study showed that strains of L.m and L.i could adhere to polystyrene and form biofilms under aerobic and microaerophilic conditions. Listeria species at 1 McFarland concentration showed that the microaerophilic conditions slow down the adhesion of most strains during the first $24 \mathrm{~h}$; however, the slowdown is followed by a relatively large development of biofilms throughout the incubation period. This behavior can be explained by the need for an adaptation time for strains to microaerophilic conditions. At the end of 


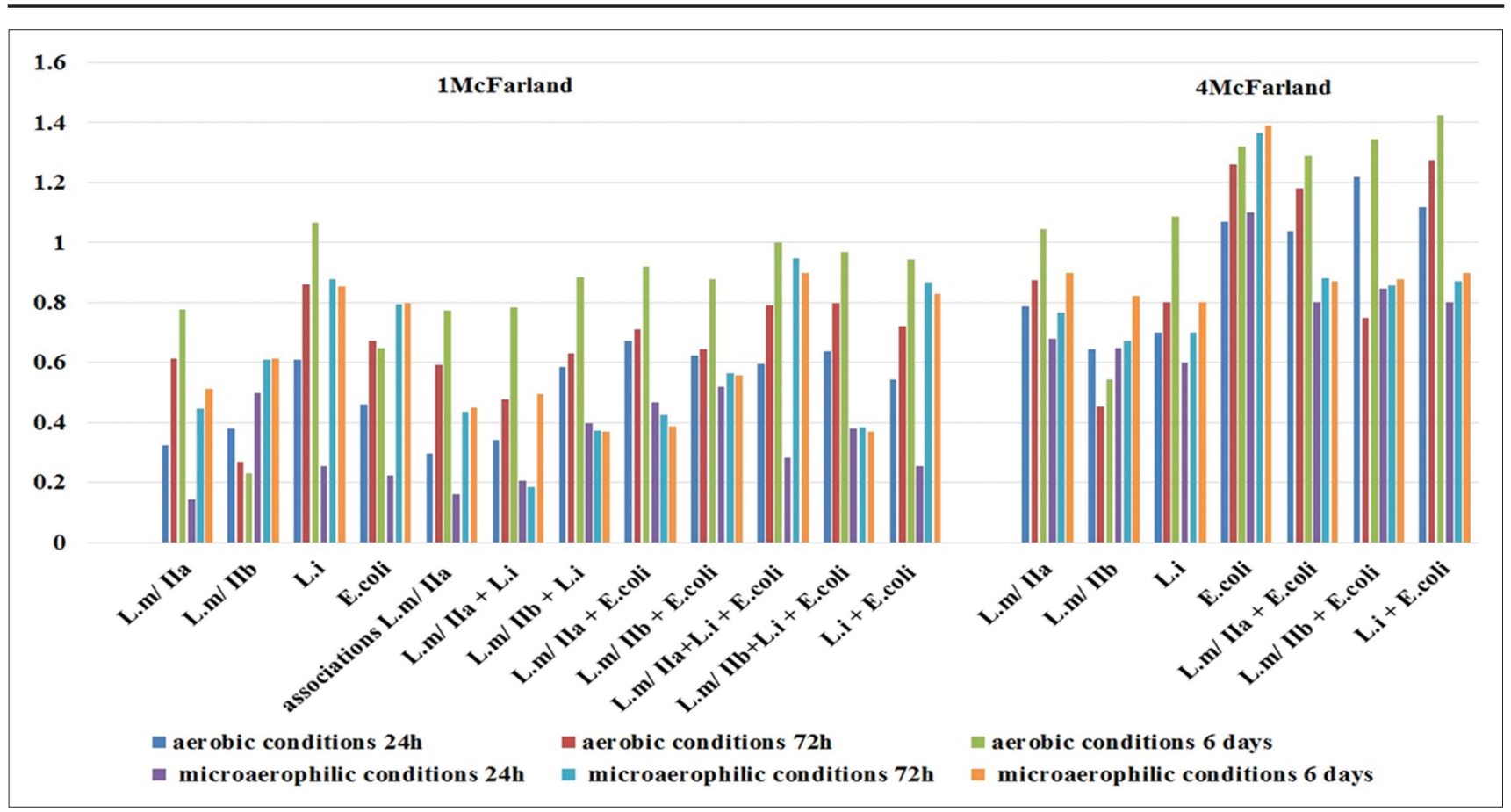

Figure-2: Effect of the incubation time on biofilms formation. Captions: L.m: Listeria monocytogenes, L.i: Listeria innocua, OD: Optical density, h: Hour.

the 6 days incubation, there was no significant difference $(\mathrm{p}>0.05)$ between the development of biofilms, whether in aerobic or microaerophilic conditions. This is probably due to the ability of Listeria to develop in atmospheres with slightly lower oxygen tension and increased carbon dioxide than air [29,30]. All L.m strains belonging to the serogroup IIa (Table-1) showed almost the same adhesion power, which led to biofilm formation after $72 \mathrm{~h}$ of incubation, except L.m 038 strain which showed at the beginning $(24 \mathrm{~h})$ of moderate adhesion. This suggests that within the same serogroup, the time spent to adapt to microaerophilic condition and biofilm formation is different.

L.m 036 strain, which belongs to the serogroup $I I b$, exhibited different behavior, developing more in microaerophilic than aerobic conditions, with less adhesion and medium biofilm formation $(\mathrm{p}<0.05)$ compared to other strains. Our results corroborate those obtained by Borucki et al. [31] and Pan [32], who found that L.m strains belonging to serogroup IIa had a higher ability to form biofilms. The study results of Kadam et al. [28] and Kalmokoff et al. [33] also showed that under certain conditions, the ability of L.m to adhere to surfaces could be related to the serogroup factor.

L.i strains showed the same adhesion power as L.m. These characteristics have been reported by Kalmokoff et al. [33], Robitaille et al. [34], and Jeon et al. [10]. They have reported the ability of L.i to adhere to several types of surfaces and their high adhesion power is similar to that of L.m strains.

The results also showed that $E$. coli strains could adhere to polystyrene and form biofilms under aerobic and microaerophilic conditions. Development was statistically better under microaerophilic conditions $(p<0.05)$. These results corroborate those of the previous studies that reported the ability of $E$. coli to form biofilms on polystyrene under different conditions [35]. However, this result differs from that reported by Auger [26], who had observed that anaerobic conditions slowed the development of biofilms considerably. This difference could be related to the fact that our tests were performed in microaerophilic and not in anaerobic conditions.

All the suspensions at 4 McFarland concentration showed a strong and early adhesion. This could be due to the high number of bacterial cells in contact with the surface, which has favored the adhesion and formation of biofilms. Microaerophilic conditions had allowed better adhesion and formation of biofilms for E. coli strains. This could be due to the ability of these strains to adapt to these conditions.

\section{Formation of multispecies biofilms}

Combinations of strains tested at both 1 and 4 McFarland concentrations, allowed the formation of strong biofilms whether in aerobic or microaerophilic conditions. This could be explained by the ability of strains to coexist in a multispecies biofilm and a probable synergy between them. In the previous studies, Jeong and Frank [36] and Chmielewski and Frank [6] reported the ability of certain pathogens to coexist within a single biofilm. The ability of L.m to grow within a multispecies biofilm has been reported by Chen et al. [37] who worked on biofilms formed by Salmonella typhimurium, STEC, and L.m and reported by Buchanan et al. [38]. The aptitude of E. coli to form multispecies biofilms has been described since 2008 [39]. Numerous examples of synergistic induction 
of multispecies biofilm formation constituted by $E$. coli and other species were reported by many studies such as Romeo [39], Giaouris et al. [40] and [41], and Larsen et al. [42]. They described the synergistic effect between different bacterial strains composing a multispecies biofilm among them L.m and E. coli. The impact of interspecies communication on biofilm development is presently not well understood [39].

Our study showed some particularities. The combination of L.m 038+ L.m 026 + L.m 043 (L.m/ IIa) showed a moderate adhesion after $24 \mathrm{~h}$ of incubation. This slow adhesion could be linked to the presence of L.m 038 strain which has already shown a moderate adhesion at $24 \mathrm{~h}$ when it has been tested alone (biofilm monospecies). The combination of L.m 023+L.m 026+L.i (L.m/IIa+L.i) has shown a moderate adhesion after $72 \mathrm{~h}$ of incubation, knowing that when tested alone, each strain showed a strong adhesion at $24 \mathrm{~h}$. This suggests that bacterial strains may show different behavior when they are simultaneously present in a multispecies biofilm, either by increasing or decreasing the adhesion capacity. This difference in behavior of L.m was reported by Giaouris et al. [41] and Jay et al. [43].

\section{Effect of the renewal of the culture medium}

The renewal of the culture medium has led to a higher biofilms development in aerobic and microaerophilic conditions. These results corroborate those obtained by Auger [26], who had noted that glucose supplementation promotes the development of biofilms. Due to its composition, TSB allowed an additional nutrient supply which likely improved the bacterial growth conditions. These results are similar to that obtained by Stepanović et al. [16], who found that L.m forms stronger biofilms in the presence of a nutrient-rich environment. Zeraik and Nitschke [44] and Azam et al. [35] showed that supplementation of culture medium with various substances influenced significantly their ability to form biofilms.

\section{Effect of incubation time}

Incubation time is an important factor in the process of biofilm formation [45]. The adhesion of the strains tested was observed after $24 \mathrm{~h}$ of incubation. The formation of biofilms was more important during the first $72 \mathrm{~h}$ of incubation to give a biofilm that tended to stabilize during the past $72 \mathrm{~h}$. Jay et al. [43] reported that biofilms of L.m could reach their maximum development after $72 \mathrm{~h}$ of incubation in optimum conditions. Our results are similar to those obtained by Han et al. [23], who showed that the mass of E. coli formed biofilms on the polystyrene surface increases with the incubation time. The previous studies of L.m biofilms have shown that after rapid initial adhesion to surfaces, bacterial populations do not increase significantly [9].

\section{Conclusion}

All the strains tested showed a more or less adhesion power depending on the variation of the studied parameters as well as an ability to form multispecies biofilms. Strains of L.m, L.i, and E. coli adhere to polystyrene and form biofilms under aerobic and microaerophilic conditions. These biofilms develop well during the first $72 \mathrm{~h}$ and are influenced by microaerophilic incubation, especially those of E. coli. Their development is even more important by renewing the culture medium and increasing the initial load of bacteria (4 McFarland).

The ability to adhere and form biofilms is different from one serogroup to another within the same species. In bacterial combination, strains and species adopt different behaviors. The ability of bacterial species to form biofilms poses a real problem in different areas. The study of the ability of strains to adhere to surfaces and to form biofilms remains complex. Several parameters can influence the adhesion, such as the nature of the surfaces, the bacterial species, the number of bacteria, the presence of a single bacterial genus or multi-genus, and many other parameters.

Recent advances in the understanding of the biofilm development cycle have indicated that, in most cases, it is a dynamic process in which factors such as nutritional conditions, temperature, oxygen tension, and osmolarity can have big influences on biofilm formation. The variation of these parameters can influence the adhesion power of bacteria as well as the extent of biofilm formation.

More researches are still necessary to understand the mechanisms of biofilm formation and the influence of different parameters in their development. The next stage of our research will involve testing the effect of some biocides on the viability of species forming biofilms and their sensitivity to antibiotics with the aim of studying the probable correlation between sensitivity toward biocides and antibiotics.

\section{Authors' Contributions}

SLA planned the study and drafted the manuscript under the supervision of LB and TMH. SLA designed the experiment protocol under the supervision of LB and TMH. SLA collected and analyzed samples did the statistical analysis and revised the manuscript under the supervision of LB and TMH. All authors read and approved the final manuscript.

\section{Acknowledgments}

We express our gratitude to bioMérieux Laboratories in Algeria for technical assistance. This study did not receive any funding.

\section{Competing Interests}

The authors declare that they have no competing interests.

\section{Publisher's Note}

Veterinary World remains neutral with regard to jurisdictional claims in published institutional affiliation. 


\section{References}

1. DuPont, G.A. (1997) Understanding dental plaque; Biofilm dynamics. J. Vet. Dent., 14(3): 91-94.

2. Parot, S. (2007) Biofilms Electroactifs: Formation, Caractérisation et Mécanismes. Thèse de Doctorat, Spécialité Génie des Procédés et de l'Environnement. p247.

3. Bougle, C. and Leroyer, R. (2003) Anti-infective coated central venous catheters: Technical aspects and clinical studies. J. Pharm. Clin., 22(3): 159-167.

4. Clutterbuck, A.L., Woods, E.J., Knottenbelt, D.C., Clegg, P.D., Cochrane, C.A. and Percival, S.L. (2007) Biofilms and their relevance to veterinary medicine. Vet. Microbiol., 121(1-2): 1-17.

5. Rodriguez, A., Autio, W.R. and McLandsborough, L.A. (2008) Effect of surface roughness and stainless steel finish on Listeria monocytogenes attachment and biofilm formation. J. Food Prot., 71(1): 170-175.

6. Chmielewski, R.A.N. and Frank, J.F. (2003) Biofilm formation and control in food processing facilities. Compr. Rev. Food Sci. Food Saf., 2(1): 22-32.

7. Chylkova, T., Cadena, M., Ferreiro, A. and Pitesky, M. (2017) Susceptibility of Salmonella biofilm and planktonic bacteria to common disinfectant agents used in poultry processing. J. Food Prot., 80(7): 1072-1079.

8. Iñiguez-Moreno, M., Gutiérrez-Lomelí, M., GuerreroMedina, P.J. and Avila-Novoa, M.G. (2018) Biofilm formation by Staphylococcus aureus and Salmonella spp. Under mono and dual-species conditions and their sensitivity to cetrimonium bromide, peracetic acid and sodium hypochlorite. Braz. J. Microbiol., 49(2): 310-319.

9. Reis-Teixeira, F.B.D., Alves, V.F. and Martinis, E.C.P. (2017) Growth, viability and architecture of biofilms of Listeria monocytogenes formed on abiotic surfaces. Braz. J. Microbiol., 48(3): 587-591.

10. Jeon, H.R., Kwon, M.J. and Yoon, K.S. (2018) Control of Listeria innocua biofilms on food contact surfaces with slightly acidic electrolyzed water and the risk of biofilm cells transfer to duck meat. J. Food Prot., 81(4): 582-592.

11. Govindaraj, D.K., Dev, K., Sadhana, R. and Jitendra, P. (2014) Comparative evaluation of factors affecting Escherichia coli biofilms on organic leafy greenwash water contact surface. J. Food Prot. Suppl., 77(1): 44-45.

12. Naito, M., Frirdich, E., Fields, J.A., Pryjma, M., Li, J., Cameron, A., Gilbert, M., Thompson, S.A. and Gaynor, E.C. (2010) Effects of sequential Campylobacter jejuni 81-176 lipooligosaccharide core truncations on biofilm formation, stress survival, and pathogenesis. J. Bacteriol., 192(8): 2182-2192.

13. Rodrigues, L.B., Santos, L.R.D., Tagliari, V.Z., Rizzo, N.N., Trenhago, G., Oliveira, A.P.D., Goetz, F. and Nascimento, V.P.D. (2010) Quantification of biofilm production on polystyrene by Listeria, Escherichia coli and Staphylococcus aureus isolated from a poultry slaughterhouse. Braz. J. Microbiol., 41(4): 1082-1085.

14. Lane, P.S., Walker, J.T. and Hunter, P.R. (2000) Microbiological Aspects of Biofilms and Drinking Water. CRC Press, Boca Raton, FL. p164-168.

15. Christensen, G.D., Simpson, W.A., Younger, J.J., Baddour, L.M., Barrett, F.F., Melton, D.M. and Beachey, E.H. (1985) Adherence of coagulase-negative staphylococci to plastic tissue culture plates: A quantitative model for the adherence of staphylococci to medical devices. J. Clin. Microbiol., 22(6): 996-1006.

16. Stepanović, S., Vuković, D., Dakić, I., Savić, B. and ŠvabićVlahović, M. (2000) A modified microtiter-plate test for quantification of staphylococcal biofilm formation. $J$. Microbiol. Methods, 40(2): 175-179.

17. Bouayad, L., Hamdi, T.M., Naim, M., Leclercq, A. and Lecuit, M. (2015) Prevalence of Listeria spp. and molecular characterization of Listeria monocytogenes isolates from broilers at the abattoir. Foodborne Pathog. Dis., 12(7):
606-611.

18. ISO. (2004) 18593-2004. Microbiology of Food and Animal Feeding Stuffs-Horizontal Methods for Sampling Techniques from Surfaces using Contact Plates and Swabs. ISO, Geneva, Switzerland.

19. ISO. (2006) 4832: 2006. Microbiology of Food and Animal Feeding Stuffs Horizontal Method for the Enumeration of Coliform Colony Count Technique. ISO, Geneva.

20. Peeters, E., Nelis, H.J. and Coenye, T. (2008) Comparison of multiple methods for quantification of microbial biofilms grown in microtiter plates. J. Microbiol. Methods, 72(2): 157-165.

21. Wang, J., Ray, A.J., Hammons, S.R. and Oliver, H.F. (2015) Persistent and transient Listeria monocytogenes strains from retail deli environments vary in their ability to adhere and form biofilms and rarely have inlA premature stop codons. Foodborne Pathog. Dis., 12(2): 151-158.

22. Lemon, K.P., Higgins, D.E. and Kolter, R. (2007) Flagellar motility is critical for Listeria monocytogenes biofilm formation. J. Bacteriol., 189(12): 4418-4424.

23. Han, R., Klu, Y.A.K. and Chen, J. (2017) Attachment and biofilm formation by selected strains of Salmonella Enterica and enterohemorrhagic Escherichia coli of fresh produce origin. J. Food Sci., 82(6): 1461-1466.

24. Stepanović, S., Vuković, D., Hola, V., Bonaventura, G.D., Djukić, S., Ćirković, I. and Ruzicka, F. (2007) Quantification of biofilm in microtiter plates: Overview of testing conditions and practical recommendations for assessment of biofilm production by staphylococci. Acta Pathol. Microbiol. Immunol. Scand., 115(8): 891-899.

25. Le Hénaff, M., Huguet, N., Richard, A., Vaillant, M., Papillon, S. and Le Gallet, A. (2016) Évaluation de l'impact de nouvelles formulations nettoyantes qui associent des dérivés de pin et des enzymes sur la réduction du biofilm microbien. Hygiènes, 24(6): 293-297.

26. Auger, M. (2012) Formation de Biofilm in vitro par des Souches d'Escherichia coli: Impacts de la Modification des Conditions Expérimentales. Thèse de Doctorat, Université de Nantes, France. p90.

27. Hassan, A., Usman, J., Kaleem, F., Omair, M., Khalid, A. and Iqbal, M. (2011) Evaluation of different detection methods of biofilm formation in the clinical isolates. Braz. J. Infect. Dis., 15(4): 305-311.

28. Kadam, S.R., Den Besten, H.M., Van Der Veen, S., Zwietering, M.H., Moezelaar, R. and Abee, T. (2013) Diversity assessment of Listeria monocytogenes biofilm formation: Impact of growth condition, serotype and strain origin. Int. J. Food Microbiol., 165(3): 259-264.

29. Renaud, J.F., Hansen, W. and Bollet, C. (1994) Manuel de Bactériologie Clinique. ASM Press, Washington, DC. p833-849.

30. Rocourt, J. (1988) The recognition and identification of Listeria species by classical methods. Turk. J. Infect., 2(4): 471-485.

31. Borucki, M.K., Peppin, J.D., White, D., Loge, F. and Call, D.R. (2003) Variation in biofilm formation among strains of Listeria monocytogenes. Appl. Environ. Microbiol., 69(12): 7336-7342.

32. Pan, Y., Breidt, F. and Kathariou, S. (2009) Competition of Listeria monocytogenes serotype $1 / 2 \mathrm{a}$ and $4 \mathrm{~b}$ strains in mixed culture biofilms. Appl. Environ. Microbiol., 75(18): 5846-5852.

33. Kalmokoff, M.L., Austin, J.W., Wan, X.D., Sanders, G., Banerjee, S. and Farber, J.M. (2001) Adsorption, attachment and biofilm formation among isolates of Listeria monocytogenes using model conditions. J. Appl. Microbiol., 91(4): 725-734.

34. Robitaille, G., Choinière, S., Ells, T., Deschènes, L. and Mafu, A.A. (2014) Attachment of Listeria innocua to polystyrene: Effects of ionic strength and conditioning films from culture media and milk proteins. J. Food Prot., 77(3): 427-434. 
35. Azam, M., Jan, A.T., Kumar, A., Siddiqui, K., Mondal, A.H. and Haq, Q.M.R. (2018) Study of pandrug and heavy metal resistance among $E$. coli from anthropogenically influenced Delhi stretch of river Yamuna. Braz. J. Microbiol., 49(3): 471-480.

36. Jeong, D.K. and Frank, J.F. (1994) Growth of Listeria monocytogenes at $10 \mathrm{C}$ in biofilms with microorganisms isolated from meat and dairy processing environments. J. Food Prot., 57(7): 576-586.

37. Chen, D., Zhao, T. and Doyle, M.P. (2015) Control of pathogens in biofilms on the surface of stainless steel by levulinic acid plus sodium dodecyl sulfate. Int. J. Food Microbiol., 207: 1-7.

38. Buchanan, R.L., Gorris, L.G., Hayman, M.M., Jackson, T.C. and Whiting, R.C. (2017) A review of Listeria monocytogenes: An update on outbreaks, virulence, dose-response, ecology, and risk assessments. Food Control, 75: 1-13.

39. Romeo, T. (2008) Bacterial Biofilms. Vol. 322. Springer Science and Business Media, New York. p293.

40. Giaouris, E., Heir, E., Hébraud, M., Chorianopoulos, N., Langsrud, S., Møretrø, T., Habimana, O., Desvaux, M., Renier, S. and Nychas, G.J. (2014) Attachment and biofilm formation by foodborne bacteria in meat processing environments: Causes, implications, role of bacterial interactions and control by alternative novel methods. Meat Sci., 97(3): 298-309.

41. Giaouris, E., Heir, E., Desvaux, M., Hébraud, M., Møretrø, T., Langsrud, S., Doulgeraki, A., Nychas, G.J., Kačániová, M., Czaczyk, K. and Ölmez, H. (2015) Intraand inter-species interactions within biofilms of important foodborne bacterial pathogens. Front. Microbiol., 6: 841.

42. Larsen, M.H., Dalmasso, M., Ingmer, H., Langsrud, S., Malakauskas, M., Mader, A., Møretrø, T., Možina, S.S., Rychli, K., Wagner, M. and Wallace, R.J. (2014) Persistence of foodborne pathogens and their control in primary and secondary food production chains. Food Control, 44: 92-109.

43. Jay, J.M., Loessner, M.J. and Golden, D.A. (2005) Modern Food Microbiology. Aspen Publishers, Gaithersburg, MD. p751.

44. Zeraik, A.E. and Nitschke, M. (2012) Influence of growth media and temperature on bacterial adhesion to polystyrene surfaces. Braz. Arch. Biol. Technol., 55(4): 569-576.

45. Bonsaglia, E.C.R., Silva, N.C.C., Júnior, A.F., Júnior, J.A., Tsunemi, M.H. and Rall, V.L.M. (2014) Production of biofilm by Listeria monocytogenes in different materials and temperatures. Food Control, 35(1): 386-391. 